\title{
LA DIMENSIÓN SIMBÓLICO - IDENTITARIA DE LA INTEGRACIÓN LATINOAMERICANA. ¿IDENTIDAD (ES) COMPARTIDA (S) MÁS ALLÁ (Y MAS ACÁ) DE LAS ASIMETRÍAS Y LA INTERGUBERNAMENTALIDAD? THE SYMBOLIC - IDENTITARY DIMENSION OF LATIN AMERICAN INTEGRATION. SHARED IDENTITY (S) BEYOND (AND BEYOND) ASYMETRIES AND INTERGOVERNMENTALITY?
}

\author{
Ernesto Dufour \\ Universidad Nacional de Lanús \\ dufour.e@gmail.com
}

\section{RESUMEN}

El presente trabajo aborda la dimensión simbólico-identitaria de la integración latinoamericana. Se trata de una puerta de entrada a la temática poco explorada por la literatura académica hegemonizada por economistas y especialistas en relaciones internacionales (RRII). Las perspectivas economicistas e institucionalistas tendieron a soslayar los aspectos culturales conforme a la preeminencia de factores políticos y comerciales. Sin embargo, la dimensión cultural en sentido amplio -entendida como el conjunto de representaciones, imaginarios e identificaciones con base territorial respecto del sí mismo tanto como del "otro" a integrar- cobra centralidad a partir de la plena vigencia de la pertenencia nacional configurada a partir del siglo XIX. De allí derivan concepciones del "interés nacional" que pueden no necesariamente confluir con un potencial "interés regional", o bien, asumir "lo regional" como mera plataforma de proyección de particularismos excluyentes de lo latinoamericano como parte constitutiva del propio proceso de formación territorial. La "colisión" de los conjuntos nacionales ampliados - cada uno de ellos con su densidad histórica, política y cultural- puede generar que las notables asimetrías estructurales, el desacompasamiento de sus sistemas políticos y la multiplicidad de las formas socio-culturales cristalizadas sean asumidos como elementos de distanciamiento y fragmentación antes que un locus de acción política mancomunada. De aquí, la necesidad de repensar a escala regional la relación entre identidad(es), (multi/trans) territorio y política(s) y las prácticas sociales, territoriales y simbólicas que los ligan, religan e imbrican. 


\section{PALABRAS CLAVES}

Integración latinoamericana, Imaginarios geográficos, Identificaciones territoriales.

\section{SUMMARY}

The present work addresses the symbolic-identity dimension of Latin American integration. It is a gateway to the subject little explored by the academic literature hegemonized by economists and specialists in international relations (RRII). Economist and institutionalist perspectives tended to ignore cultural aspects according to the pre-eminence of political and commercial factors. However, the cultural dimension in a broad sense - understood as the set of representations, imaginaries and identifications with a territorial basis with respect to the self as well as the "other" to be integrated - acquires centrality from the full validity of the national belonging configured to from the nineteenth century. From there derive conceptions of "national interest" that may not necessarily converge with a potential "regional interest", or assume "the regional" as a mere platform for projection of particularisms that exclude Latin America as a constituent part of the territorial formation process itself. The "collision" of the extended national groups - each one of them with its historical, political and cultural density - can generate that the notable structural asymmetries, the discomparison of their political systems and the multiplicity of the crystallized socio-cultural forms are assumed as elements of distancing and fragmentation before a locus of joint political action. Hence, the need to rethink on a regional scale the relationship between identity (s), (multi / trans) territory and politics (s) and the social, territorial and symbolic practices that link, link and overlap.

\section{KEYWORDS}

Latin American Integration, Geographic Imaginaries, Territorial Identifications. 
El presente trabajo aborda la dimensión simbólico-identitaria de la integración latinoamericana. Se trata de una puerta de entrada al tema de la integración poco frecuentada por la literatura académica, hegemonizada por los desarrollos teóricos provenientes, principalmente, del campo de las relaciones internacionales.

Esta disciplina toma a los procesos de integración regional como objeto primario de estudio tanto en sus enfoques realistas como idealista, este último de fuerte matriz liberal y ambos inscriptos en el paradigma del racionalismo occidental.

Dicho paradigma toma la experiencia de la Unión europea como modelo a seguir por parte de los proyectos de integración regional a lo largo $\mathrm{y}$ ancho del mundo con un fuerte carácter prescriptivo. A estos enfoques se le agregan luego los parámetros epistemológicos del constructivismo, autoasumido como un enfoque superador de las tradiciones anteriores.

Tales estudios predominantes oficiaron de marcos de referencia preferencial de las acciones gubernamentales en la instancia regional configurada por los diferentes proyectos integradores latinoamericanos como ALALC, ALADI, Mercosur y CAN.

Desde el interior de esta perspectiva, se identifican como factores centrales que motorizan la integración -siempre con la experiencia de la Unión europea como telón de fondo- a:

1) La necesidad de ampliar los mercados nacionales.

2) El diseño de instituciones regionales fuertes.

Estas perspectivas de tipo economicista e institucionalista se toparon con dos límites estructurales. Por un lado, las asimetrías de las distintas economías a integrar y, por otro, la imposibilidad de alcanzar estadios de supranacionalidad que implican la cesión de soberanía por parte de los estados a instancias regionales superiores.

Frente a estos constreñimentos duros, las instituciones regionales fueron en la práctica concreta, reducidas a meros organismos intergubernamentales, sin presupuestos significativos, sin poder de decisión politica ni incidencia territorial efectiva, deviniendo así meras 
"sopinhas de letras" (Martin, 1993), sin encarnadura en los millones de ciudadanos latinoamericanos de a pie.

Por su parte, la perspectiva constructivista entiende que tales obstáculos pueden ser superados a partir de "procesos cognitivos" que incluyen prácticas simbólicas de interacción social que permiten modelar identidades e intereses, tanto en las dirigencias como en el conjunto de las sociedades involucradas en pos de objetivo de construir una "cultura de la integración" que allane el camino de la misma.

El objetivo de este trabajo consiste en una doble interpelación o revisión crítica a esta concepción predominante, de fuerte matriz eurocéntrica, desde la perspectiva del pensamiento situado o nacionallatinoamericano y a partir de dos vertientes intelectuales:

- Por un lado, desde los dispositivos teóricos de la geografía contemporánea, específicamente en su giro político y cultural o la llamada "geografía de los imaginarios" (Lindón y Hiernax, 2012).

- Y por otro, desde el vasto legado de unidad latinoamericana, muy particularmente la obra de Manuel Ugarte. El primero de nuestros pensadores en advertir acerca de la necesidad de forjar un nacionalismo a escala latinoamericana como forma de defensa efectiva de nuestras soberanías culturales, políticas y territoriales frente al emergente poder norteamericano, a inicios del XX.

Manuel Ugarte, propone en el momento que la instauración del orden oligárquico y sus patrias chicas consecuentes se consolidan, un "salto de escala" en la imaginación geográfica y en los sentidos de pertenencia territorial. Asimismo, se ensaya aquí una interpelación mutua y recíproca entre ambas vertientes.

En principio, dos aspectos a problematizar de los enfoques predominantes. Por un lado, estos abordajes dejan de lado las relaciones de poder real involucradas en los procesos de integración que operan por detrás y través de los diseños institucionales y, por otro, la reducción de toda la complejidad multidimensional inherente, propia de la realidad territorial de la cual son parte y pretenden incidir. 
La dimensión simbolico-identitaria de la integración latinoamericana si bien no es desconocida, en alguna medida, aparece como un aspecto tangencial, secundario o epifenoménico respecto de los factores explicativos predominantes antes mencionados.

Los aspectos culturales fueron históricamente soslayados conforme a la preeminencia de imperativos políticos y económicos de primer orden. Tales urgencias descansaban en el supuesto que frente a la necesidad de ampliar el mercado y de adquirir mayor poder de negociación en la escena internacional, "lo cultural" aparecía como una cuestión dada, conforme a la herencia colonial compartida y a una misma raíz lingüística y religiosa. La cuestión no representaba un eje nodal a trabajar a nivel programático más allá de la promoción de una mayor difusión de expresiones artísticas o culturales latinoamericanas.

Por añadidura, se asumía que dadas ciertas condiciones políticoinstitucionales y materiales la identificación de los pueblos y sociedades con el proceso integrador emergería de manera más o menos espontánea.

Se trata de una cuestión intensamente vivida pero, en alguna medida, mal identificada y poco procesada todavía. Como si la problemática de la integración se agotara en la necesidad de ampliar el mercado o en la ingeniería de los diseños institucionales, o bien, desde otro punto de partida, en la sola "puesta en valor" discursiva del vasto legado cultural de unidad continental.

Sin embargo, la dimensión simbólica en sentido amplio -entendida como el conjunto de representaciones nacionales y regionales, imaginarios geográficos e identificaciones de base territorial, "lealtades" respecto del sí mismo como del "otro" latinoamericano, ahora a integrar-; cobra centralidad a partir de la plena vigencia de las llamadas por el pensamiento nacional-latinoamericano identidades de patria chica configuradas a partir del advenimiento del orden oligárquico desde mediados del siglo XIX, más allá de sus eventuales erosiones, resignificaciones y/o reapropiaciones.

De allí derivan modos de concebir "lo regional" como mera plataforma de proyección de particularismos de tipo endogámico, estructurados a escala nacional en sentido restringido. Esto es, excluyentes de "lo latinoamericano" como parte constitutiva -0 fundante- de la propia formación territorial. 
El problema remite a las representaciones y sentidos internalizados respecto de "nuestra propia nación" y de las otras ahora a integrar. Esto es, "ser argentino", "ser brasileño" incluye ¿"ser latinoamericano?". Borges dijo una vez en el marco de una entrevista, sagaz y cínicamente, que había conocido a muchos peruanos, brasileños y colombianos "pero nunca a un latinoamericano" porque "nadie piensa ni se siente como latinoamericano". Esto es realmente así en la medida que nadie, o muy pocos, se reconocen como tal. Sentidos de pertenencia internalizados que ofician de marco perceptivo para las prácticas políticas, sociales y culturales en la instancia regional, tendientes tanto a la ratificación de las formaciones territoriales heredadas. Esto es, el "status quo" territorial, tal como lo conocemos, que asume a "América Latina" como un mero referente geográfico compuesto por la sumatoria de una treintena de países, a modo de pacht-work. O bien, hacia su reconfiguración en pos de una nueva realidad territorial, directamente vinculada con la emergencia, profundización -o relanzamiento- del proyecto de unidad continental, más allá de esfera comercial o de cooperación diplomática.

Desde esta perspectiva de nacionalidad restringida o de patria chica, la "colisión" de los conjuntos nacionales ampliados puede generar que las notables asimetrías estructurales de sus economías, los diferentes legados históricos de sus sistemas políticos y la heterogeneidad de las formas socioculturales cristalizadas sean asumidas como elementos de distanciamiento y fragmentación antes que campos críticos de acción mancomunada. Valgan como ejemplos sintomáticos, el conflicto entre Argentina y Uruguay en torno a la instalación de la planta papelera Botnia, años atrás, el reclamo histórico de Bolivia por el acceso al litoral marítimo, las quejas en Brasil ante la nacionalización de la Petrobrás por parte del gobierno de Evo Morales o las, más recientes, amenazas de escalada bélica de Colombia hacia Venezuela y el rechazo de migrantes venezolanos en la frontera con Brasil, entre muchos otros.

Si bien los recelos y disputas de patria chica no alcanzan una rivalidad extrema, de tipo hobbesiana, parecen tener la fuerza suficiente para erosionar y despotenciar cualquier intento integrador que vise trascender los parámetros del regionalismo abierto de matriz neoliberal y el vínculo político apenas intergubernamental.

1 Disponible en: www.unla.edu.ar/greenstone/collect/archived/index/assoc/HASH017b/ad035185.dir/ doc.pdf 
En definitiva, ¿es posible concebir algún tipo de unidad política continental sin la emergencia de un demos latinoamericano? O lo que es lo mismo, sin un sentido de pertenencia compartido. "Sentido" entendido como algo más que mero significado o argumentación teórica o ideológica sino como la unión fecunda entre sentir y pensar. Lo cual incluye un hacer consecuente.

El carácter multidimensional de los procesos regionales excede por mucho el rol de las cancillerías, grupos económicos, redes societarias e incluso, la eventual sintonía ideológica y personal de los mandatarios y mandatarias de la región o "diplomacia presidencial", característica del político período precedente. Los indudables avances de los proyectos de UNASUR, MERCOSUR, ALBA (ahora en camino de ser dramáticamente obturados) no necesariamente derivan en un sentido de pertenencia compartida, en el plano de la estructura de sentimientos o habitus de las mayorías latinoamericanas. De la misma manera, no es posible alcanzar dicha identificación regional apelando -únicamente- a un latinoamericanismo abstracto de carácter testimonial o retórico, sin encarnadura en la experiencia vital de millones de latinoamericanos de a pie.

Este trabajo parte de la siguiente premisa ético- política: la eventual profundización o relanzamiento de la integración latinoamericana más allá de la esfera comercial y alcanzar un mayor grado de unificación política y productiva debe incluir como prerrequisito cohesionante el involucramiento activo de anchas bases populares (Felipe Herrera, 1967). Una visión ético-estratégica que el pensamiento nacional- latinoamericano viene remarcando desde Ugarte a esta parte -por no remontarnos a los procesos independentistas- y surgida en el plano de la acción y el ensayo político, no de los ámbitos y saberes académicos. Es notable en este sentido, la conferencia dictada por Juan Domingo Perón en la escuela de defensa en noviembre del 1953 sobre la unidad continental ${ }^{2}$. Dicha conferencia tiene la virtud de pasar del plano ideacional, configurado en la obra de Manuel Ugarte, al nivel programático al resaltar que la unidad de los países latinoamericanos no puede escindirse de la integración de los pueblos. Y esto implica sentirnos parte de un mismo colectivo como resultado de un trabajo de acción política efectiva de carácter territorial, esto es, con

2 Disponible en: http://constitucionweb.blogspot.com/2010/04/unidos-o-dominados-discurso-de-peron-en. html 
capilaridad y penetración en las espacialidades cotidianas o espacio banal (Milton Santos, 1996).

En definitiva, el sujeto de la integración no puede ser otro más que los pueblos a través de la única entidad dotada de legitimidad soberana, los estados nacionales -no el mercado ni solo las redes sociales- piezas centrales en la estructuración del sistema mundial a pesar de los intentos globalizadores por reducirlo a un carácter de entidad fallida en las periferias del mundo. A partir de un juego de doble pinzas, el estado-nación reactualiza incesantemente su carácter decisivo bajo nuevas coordenadas del campo de relaciones de fuerza en pugna. Por un lado, en los centros de poder mundial el estado motoriza y operacionaliza su proyección estratégica en el entramado disputas en ciernes. Por otro, en las periferias, su atributo normativo -emanado de su condición soberana- deviene factor nodal que a ser "capturado" en tanto elemento facilitador de la reproducción y despliegue de las determinaciones de poder global, al tiempo que instancia de confrontación política y espacio de condensación del conflicto social a escala local.

En América Latina, a diferencia de Europa, el pasaje del estado colonial al estado nacional requirió de la creación, por parte de las elites políticas y culturales, de una entidad político-cultural-geográfica al momento inexistente, la nación. Como destacan Ansaldi y Giordano (2012), las regiones tenían existencia previa a la nación la cual se superpuso a las formaciones territoriales preexistentes. Pero esta superposición no se desplegó sobre "tabula rasa" sino, antes bien en "amalgama áspera" debido a las -de acuerdo a la metáfora de Milton Santos (1996)- rugosidades propias de las formas espaciales heredadas o materialidad cristalizada de ordenes políticos pretéritos con toda su densidad histórica y cultural. Como destaca Fermín Chávez (2012), en el periodo precedente existía un fuerte sentido comunidad compartida pero expresado a otras escalas. La patria era la "patria americana", espontáneamente imbricada a las identificaciones locales arraigadas.

Los estudios académicos recientes sobre el estado en américa latina permiten romper con su noción naturalizante y el carácter supuestamente universal que le asigna un comportamiento teleológico (es decir, pre-político) o ineluctable fundado en la experiencia europea. La ruptura de la visión jurídico-normativa predominante en el campo académico, constreñida a la 
formalidad de tipo institucionalista y la reificación del estado que conlleva, posibilita incorporar al análisis dimensiones involucradas en su proceso de formación que fueron soslayadas o invisibilizadas y que dan cuenta de la complejidad sociopolítica y territorial inherente al proceso. Desde esta perspectiva, es posible resaltar que por tras - y a través-de la "forma estado", con todo su conglomerado burocrático y jurídico-administrativo, lo que opera es la cristalización de un orden político, vale decir, determinaciones de poder "triunfantes" encarnadas en actores sociales concretos, frente a otros proyectos alternativos de ordenamiento de las relaciones sociales, económicas y culturales. "La administración burocrática es la forma más racional de ejercer una dominación" (Weber, 2002, p. 224).

De esta manera, el estado en américa latina es resultante de un proceso de disputas sociohistóricas territorializadas que emerge como espacio de conflictividad. Y esas disputas no son otras que aquellas generadas por la cristalización del orden capitalista periférico concomitante con el surgimiento del imperialismo que resultó en la consolidación de regímenes formalmente soberanos de cuño oligárquico en tanto forma de dominación político-social propia del modelo primario-exportador ensamblado al orden mundial configurado por gran Bretaña desde mediados del siglo XIX.

De acuerdo a Netl (en Oszlak, 1982), la internalización de la identidad nacional -entendida como el control ideológico de la dominación- es atributo central de estatalidad en paridad de jerarquía respecto de los restantes atributos del modelo propuesto por el autor, a saber externalización del poder, institucionalización de la autoridad y diferenciación del control. Lo "identitario" no aparece entonces como mero anexo "superestructural" o epifenómeno de los restantes atributos "duros" sino como elemento en la legitimación del orden, en el plano simbólico e ideacional en tanto requisito fundamental para la existencia y reproducción de la forma estado. Orden de poder metabolizado, "hecho cuerpo", a través de la identidad nacional de él derivada. Un "hacer cuerpo" que nunca es pasivo o inerme sino que se encuentra en incesante proceso de apropiación y reapropiación. Así, las identidades como expresión orden político que las originó, en un nivel ontológico, más allá -y más acá, (más acá en el sentido de la propia intimidad)- de las categorías políticas e institucionales en sentido estricto. El cuerpo, acaso, como el primer territorio.

Hoy día, todos nos asumimos argentinos, uruguayos, ecuatorianos, venezolanos, etc. Uno no puede decirle, sin más, a un uruguayo por 
ejemplo, que lo que él siente como propio, su identidad nacional uruguaya es en realidad producto de una manipulación histórica por parte del poder imperial británico en el siglo XIX. En términos ontológicos, su pertenencia territorial es legítima, es porque está.

Ahora bien, ¿puede la institución Estado forjar algo para lo cual no está hecho? El punto es que no existe algo parecido a un "estadoregión" equivalente al estado-nación con fuerza heurística y atribuciones legitimantes capaces de configurar una "identidad regional" consecuente. Pareciera que estamos en un atolladero. Entre otras cosas porque ese "salto de escala" no refiere a una mera cuestión de proporcionalidad sino que involucra un conglomerado de complejidades y sedimentaciones culturales e identitarias que bregan por ratificar las formaciones territoriales heredadas. Esto es, las de las patrias chica.

Ahora bien, la "crisis existencial" (Sanahuja, 2012) de la Unión Europea -aquel constructo que nos fuera presentado como paradigma a seguir-es elocuente en este sentido debido a la profunda brecha evidenciada entre las ciudadanías europeas y toda parafernalia tecnocrática de Bruselas. No solo la política continua allí estructurada a nivel nacional sino también los sentidos de pertenencia permanecen arraigados en ese nivel a pesar de los profusos intercambios laborales y educativos de millones de europeos promovidos por políticas activas de la Unión Europea.

Llegados a este punto, existen cuestiones epistemológicas a considerar, a modo de precuaciones de método. Primero, la identidad no se construye solo a partir de un mayor conocimiento cultural acerca del otro, como cree el constructivismo ingenuo. En principio porque "lo simbólico" es expresión eufemística de relaciones de poder (Bourdieu, 1999). Es un poder subordinado a estructuras de relaciones de fuerza no ya simbólicas sino estrictamente reales " (...) haciendo esconder la carga de violencia que encierran [las palabras] objetivamente y transformándolas así en poder simbólico, capaz de producir efectos reales sin gasto aparente de energía" (Bourdieu, 1999, p. 73). Estamos en presencia de la politización de la cultura y la identidad, que aparecen ya no más como mero ropaje simbólico u ornamental respecto del poder en sentido lato sino como el expresión en sí mismas de esas relaciones de poder en un registro cultural. El poder como su dimensión constitutiva. No hay proyecto de poder sin lógica cultural. 
De acuerdo al pensamiento nacional-latinoamericano, a partir de la obra de autores como Abelardo Ramos, Fermín Chávez y Arturo Jauretche, el papel de la cultura en los países semicoloniales adquiere un papel crucial a diferencia de las colonias donde el dispositivo de dominación es el ejército. La cultura adquiere un carácter político preponderante a través de aparatos culturales de colonización pedagógica básicamente la escuela, la prensa, los intelectuales y su literatura, las academias y la universidad en tanto usinas de producción y reproducción de saberes funcionales al orden político imperante. De aquí la necesidad de diferenciar entre el nacionalismo de los países dominantes y el nacionalismo de los países dominados. La disputa cultural por el sentido, el alcance y la defensa de lo nacional, con todas sus implicancias deviene en américa latina un locus de la acción política emancipatoria.

En segundo término, es necesario remarcar la distinción crucial entre cultura e identidad que muchas veces aparecen como equivalentes y no necesariamente lo son a partir de la perspectiva de Alejandro Grimson (2011). Lo primero refiere a configuraciones culturales, por ejemplo "el Tango". E identidad a los sentidos de pertenencia colectiva. Personas de distintos países pueden aprender a bailar tango pero no por eso sentirse parte de un mismo colectivo.

El constructivismo tuvo la virtud de resaltar que los objetos sociales no tienen un origen divino o natural sino que son resultado de lo que hacen los hombres y mujeres. Pero detener la reflexión en esa premisa deja de considerar que, a pesar de ese origen construido, las cosas del mundo no son meros "juegos de sentido" o "ficciones orientadoras" impuestos de arriba hacia abajo por parte de elites intelectuales, sino que existen internalizaciones y reapropiaciones populares que los metabolizan desde su propio acervo cultural, modificándolos, de una manera que no puede ser predeterminada.

Así, el "constructo", en caso de sedimentarse, pierde su condición de tal para devenir en realidad ontológica. Se hace cuerpo y comienza a formar parte de la cotidianeidad. En rigor, los sentidos de pertenencia no son ni esencias ni constructos sino sedimentos. La imagen de sedimento implica movimiento y, al mismo tiempo, cierta estabilidad, siempre provisoria y plausible de modificarse de acuerdo a los avatares de los fijos y flujos 
(materiales e ideacionales) del entorno donde interinamente reposa. Somos -o vamos siendo- aquello que va decantando.

Los sentidos de pertenencia, inescindibles de la condición humana al estar presentes de manera tácita o explícita -tanto da- en el devenir de lo humano, permite vislumbrar el pasaje de la concepción de cultura como objeto, práctica o actividad, propia del paradigma occidental hacia la cultura asumida, desde la cosmogonía del pensamiento de Rodolfo Kusch, desde el estar-siendo. Dice el autor:

Una cultura americana no ha de consistir en ver alguna vez un cuadro y decir que ese cuadro es americano. Lo americano no es una cosa. Es simplemente la consecuencia de una profunda decisión por lo americano entendido como un despiadado aquí y ahora y, por ende, un enfrentamiento absoluto consigo mismo. ( Kusch, 1976, p.71)

Desde esta mirada, permanece aún latente una potencial capa de pertenencia territorial a escala latinoamericana, a modo de nueva sedimentanción. Una entidad discursiva, Latinoamérica, que hasta el momento, se manifiesta en un registro polisémico de tipo geográficoidiografico, turístico, literario, fólclorico, o bien, ético-politico, ideológico o geocultural pero que -en todos los casos- no termina de arraigar en un plano identitario. América Latina parece más bien pivotear en una incesante apuesta liminar por constituirse como lugar de pertenencia siempre inconcluso. "Nem vá nem fica, fica sempre em estado de poesia", como dice la canción del músico brasileño Chico César.

Desde esta perspectiva, la obra de Ugarte emerge como un salto en la imaginación territorial del sentido de nacionalidad en clave continental, a modo de nueva reapropiación. Una reinterpretación del sentido de pertenencia, intrínsecamente ligado a un territorio, que es concreto e ideacional a la vez, justo en el momento en que comenzaba a sedimentarse las nacionalidades de patria chica de cuño oligárquico. Su concepción surge como consecuencia directa de la emergencia del imperialismo norteamericano a inicios del XX.

Ahora bien, es interesante el desafío de la obra de Ugarte para repensar la actual realidad latinoamericana a inicios del siglo XXI. Al igual que en tiempos de Ugarte, vivimos un contexto de dramática reconfiguración del 
orden mundial, ahora con la emergencia de un bloque de poder conformado por China y Rusia que pone en cuestión la hegemonía norteamericana en el comando del capitalismo global. Contexto en que EEUU, no obstante -o mas bien por causa de ello- se reposiciona ferozmente en su "patio trasero".

Estamos en presencia de una nueva correntada de poderes mundiales que remueven sedimentaciones identitarias. Ninguno de los desafíos que atraviesa la región puede ser abordado políticamente desde la soledad de nuestras patrias chicas. Esto es válido aún para Brasil que -con todo- no deja de ser vulnerable frente a los poderosos del mundo. Exigen respuestas continentales, que es la escala real de intervención de los poderes fácticos mundiales.

El relanzamiento del sentido de pertenencia latinoamericana bajo nuevas coordenadas aparece como ámbito "inédito" de la acción politica emancipatoria en un contexto en el que el orden globalitario -siguiendo a alemán (2016) - disputa no solo las esferas de la economía y la política sino el propio campo del sentido, de la representación y de la producción biopolítica de la subjetividad. El orden globalizador aparece como una fenomenal fábrica de subjetividad que visa formatear las "capas tectónicas" de nuestra intimidad, constitutivas del propio sujeto, en un nivel ya no solo ideológico, sino ontológico, ligado al ser y al estar. Los propios sujetos somos capturados en la lógica de reproducción del poder de las corporaciones, los medios de comunicación y las redes tecno-digitales que han tomado el botín más valioso como nunca antes, el mundo de la subjetividad y sus sentidos.

De acuerdo a Alemán (2016), el neoliberalismo es la primera formación histórica que trata de tocar la propia constitución del sujeto, tocar su núcleo ontológico. Un estadío más allá de la llamada hegemonía política y cultural. Como decía Margareth Tacher "nuestro método es el mercado nuestro objetivo el alma".

El relanzamiento de la nacionalidad a partir del redescubrimiento de nuestra propia latinoamericaneidad en tanto instancia vital que nos permite "respirar juntos" ante los peligros en ciernes. En este marco de ideas, América Latina no es estrictamente un lugar sino un entrelugar (Antelo, 2014) que aparece ante nuestras patrias chicas "desvencijadas" 
-o escangalhadas- como posibilidad de un espacio existencial que nos permita "tomar aire" frente al tsunami del orden globalitario.

Por tanto, no se trataría de complementar a nuestra identidad nacional sedimentada una eventual y abstracta "identidad latinoamericana" y, mucho menos, reemplazarla por otra sino resignificar y reapropiarnos de las premisas ontológicas que fundan nuestra pertenencia bajo nuevas coordenadas. No se trata de adosar nada nuevo sino de -al modo ugartianoreconocer y abrazar los pilares constitutivos de nuestra propia formación, que es territorial y personal a la vez, y que es inescindible de la región desde la cual surgimos, somos parte, nos atraviesa y constituye, queramos o no.

Una potencial pertenencia latinoamericana que se explica más que como extensión o complemento de la propia identidad como imbricación con el "otro latinoamericano"; entendido como aquello que esta fuera de mí y, sin embargo, me constituye en lo mas mío, en mi condición de brasileño, argentino, colombiano, peruano, uruguayo, etc.

En esta clave de lectura puede ser visto el film argentino Las Acacias del año 2011. El film relata el vínculo que se establece entre un camionero del conurbano bonaerense, solitario y apesadumbrado por el paso de los años y una mujer paraguaya con su pequeña hija en brazos que debe llevar a Buenos Aires, a pedido del patrón, en su camión en el que transporta madera desde Paraguay. Casi no hay diálogo y priman los silencios tensos y pesados. Las distancias sociales y simbólicas junto a los recelos nacionales entre ambos mundos son una barrera infranqueable. Sin embargo, el vínculo -a fuerza de realidad por las necesidades mutuas y la proximidad física que impone la cabina- los va cercando a los tres a medida que se desplazan por la ruta. Es notable como el camionero se encuentra a sí mismo solo a través del encuentro, sensible y afectivo, con aquello -en principio- tan ajeno, encarnado en la mujer paraguaya y su bebé que, sin embargo, lo convoca, interpela y constituye, incluso a pesar de él mismo. Atraviesan no solo fronteras y kilómetros sino también sedimentaciones culturales en ese punto en que ya no refieren a símbolos, representaciones o -ni siquiera-a identificaciones sino al -siguiendo a Rodolfo Kusch (1976)- así de la $v i d a$, el mero estar, incesantemente habitado por multitudes y soledades. El pulsar de la vida, sin mistificación. 
No hay otra forma de ser latinoamericano sino siendo lo que ya somos o venimos siendo. Somos latinoamericanos porque somos argentinos, brasileños, ecuatorianos, paraguayos, etc. Y somos argentinos, brasileños, paraguayos, ecuatorianos, etc. porque somos latinoamericanos. Lo latinoamericano como aquello que permite reconocerme, autoafirmarme y, en un futuro inminente, -quizás- autorealizarme.

El punto nodal, entonces, para la acción política emancipatoria y el relanzamiento de la unidad continental bajo nuevas coordenadas históricas y geopolíticas radicaría en dilucidar el modo de pasar del reconocimiento -e incluso la celebración- del vasto caleidoscopio cultural latinoamericano (he aquí el límite del constructivisno y el multiculturalismo) al relanzamiento de sentidos de pertenencia compartidos, ese despiadado enfrentamiento consigo mismo, aquí y ahora.

Remedios de Escalada, Argentina, 2018.

\section{BIBLIOGRAFÍA CONSULTADA}

Alemán, J. (2016). Horizontes neoliberales en la subjetividad. Olivos: Grama ediciones.

Ansaldi,W., y Giordano,V. (2012). Presupuestos teóricos-metodologicos para el análisis socio-histórico del proceso de formación de los estados latinoamericanos. Revista Estudios del ISHiR-CONICET, Argentina. Disponible en: http://www.revista.ishir-conicet.gov.ar/ index.php/revistaISHIR| Año 2, Número. 4, 2012.

Antelo, R. (2014). Imágenes de América Latina. Saenz Peña: Eduntref.

Bourdieu, P. (1999). Sobre el poder simbólico en Intelectuales, política y poder. Buenos Aires: Eudeba, 1977.

Chávez, F. (2012). Epistemología para la periferia. Jaramillo, Ana (Comp.) Remedios de Escalada: Ediciones de la UNLa.

Galasso, N. (2012). Manuel Ugarte y la unidad latinoamericana. Buenos Aires, Ediciones Colihue.

Grimson, A. (2005). Fronteras, estados e identificaciones en el Cono sur. En Cultura, política y sociedad Perspectivas latinoamericanas 
(antología) Daniel Mato (comp.) Colección Grupos de Trabajo. Buenos Aires: CLACSO.

Grimson, A. (2011). Los límites de la cultura. Crítica de las teorías de la identidad. Buenos Aires: Siglo XXI Ed.

Haesbaert, R.(2014). VivirnoLimite. Territorioemulti/transterritorialidades em tempos de in-segurança e contenção. Rio de Janeiro: Bertrand Brasil.

Herrera, F. (1967). Nacionalismo latinoamericano, Santiago de Chile: Universitaria.

Jameson, F. (2012). Posmodernismo: la lógica cultural del capitalismo avanzado. Vol. I. Buenos Aires: La marca editora, 1997.

Jauretche, A. (1968). Manual de Zonceras Argentinas. Buenos Aires: Peña Lillo editor.

Kusch, R. (1976). Geocultura del hombre americano. Buenos Aires: Fernando García Cambeiro Ed.

Lindon, A. y Hiernaux, D. (2012). Geografias de lo imaginario. Barcelona: Anthropos Editorial ; México: Universidad Autónoma Metropolitana - Iztapalapa. Div. Ciencias Sociales y Humanidades.

Oszlak, O. (1982) Reflexiones sobre la formación del Estado y la construcción de la sociedad argentina. Desarrollo Económico Revista de Ciencias Sociales, Vol. XXI, 1982, Enero-Marzo: Buenos Aires, Argentina.

Ramos, A. (2011). Historia de La Nación Latinoamericana. Buenos Aires: Editorial Continente.

Ramos, A. (2012). Revolución y Contrarrevolución en la Argentina. Buenos Aires: Editorial Continente.

Sanahuja, José Antonio (2012). Las cuatro crisis de la Unión Europea. En Manuela Mesa (coord.) Cambio de ciclo: crisis, resistencias y respuestas globales. Anuario 2012-2013. Madrid: CEIPAZ-Fundación Cultura de Paz.

Santos, M. (1996). A natureza do espaço. Técnica e Tempo. Razão e Emoção. São Paulo: Hucitec.

Ugarte, M. (1953). El porvenir de América Latina. Buenos Aires: Editorial Indoamérica. 
Ugarte, M. (1960). La Patria Grande. Buenos Aires: Ediciones Coyocán, 2da edición.

Ugarte, M. (1962). El destino de un continente. Buenos Aires: Ediciones de la Patria Grande, 2da edición.

Weber, M. (2002). Economía y sociedad. Esbozo de sociología comprensiva. Madrid: FCE.

Wortman, A. (2009). Cultura y Estrategia para el Mercosur. Buenos Aires.: Ficha Seminario DPPIR FLACSO, 